Celgene

\title{
In Multiple Sclerosis
}

\section{GREY MATTERS, TOO}

Think White and Grey to Complete the Picture

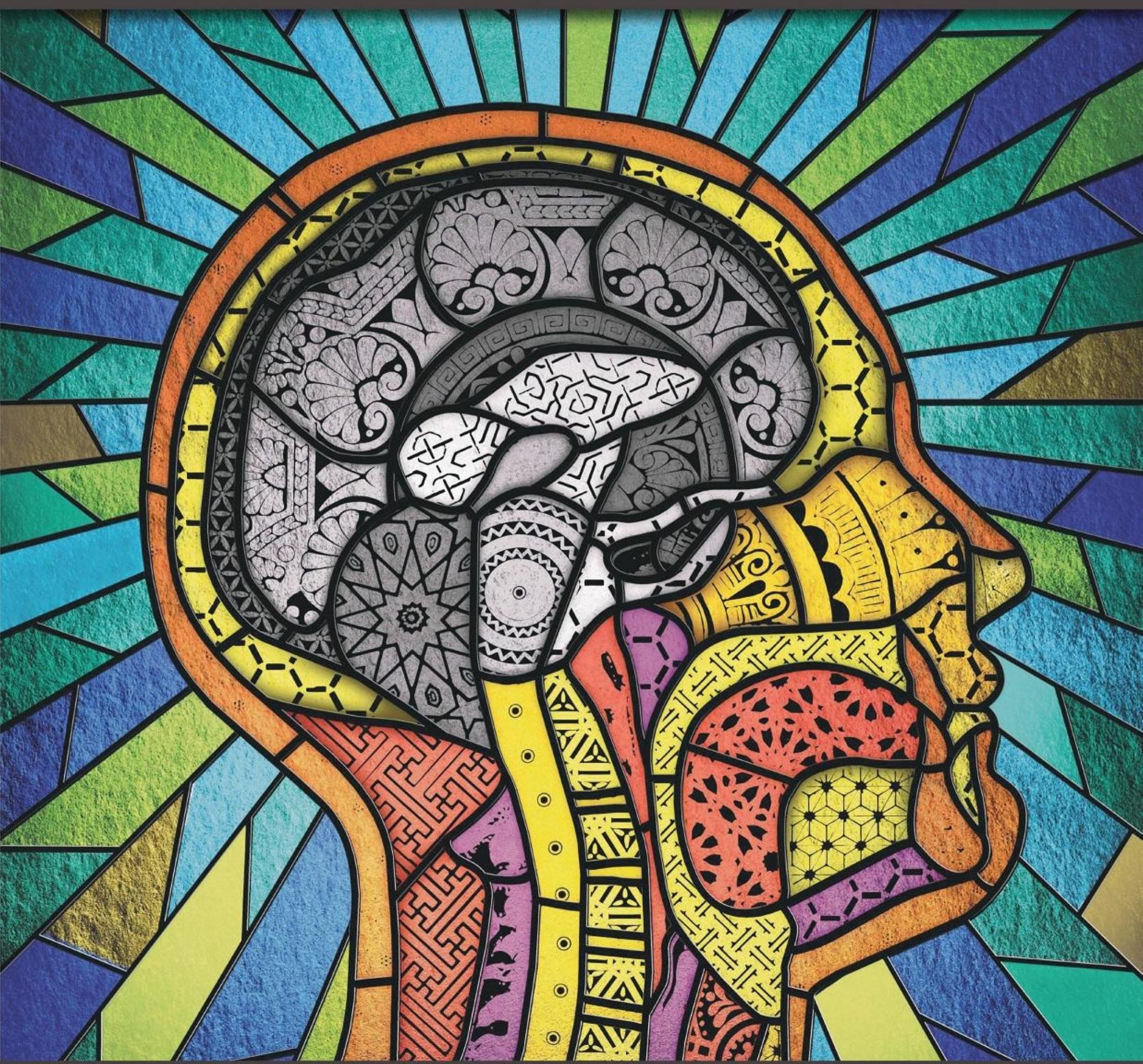

Visit MSGreyMatters.com for more information. 


\title{
Comorbidity in multiple sclerosis: its temporal relationships with disease onset and dose effect on mortality
}

\author{
I. J. Chou ${ }^{a, b, c}$ (iD, C. F. Kuo d,e , R. Tanasescu, ${ }^{a, f}$, C. R. Tench ${ }^{a}$, C. G. Tiley ${ }^{g}$, C. S. Constantinescu ${ }^{a}$ (iD) and \\ W. P. Whitehouse ${ }^{b *}$
}

\begin{abstract}
${ }^{a}$ Division of Clinical Neuroscience, School of Medicine, University of Nottingham, Nottingham; ${ }^{\mathrm{b}}$ Division of Academic Child Health, School of Medicine, University of Nottingham, Nottingham, UK; ${ }^{\mathrm{c}}$ Division of Paediatric Neurology, Chang Gung Memorial Hospital,

Taoyuan, Taiwan; ${ }^{\mathrm{d}}$ Division of Rheumatology, Orthopaedics and Dermatology, School of Medicine, University of Nottingham, Nottingham, UK; ${ }^{\mathrm{e}}$ Division of Rheumatology, Allergy and Immunology, Chang Gung Memorial Hospital, Taoyuan, Taiwan; ${ }^{\mathrm{f}}$ Division of Neurosciences, Department of Neurology, Colentina Hospital, University of Medicine and Pharmacy Carol Davila, Bucharest, Romania; and ${ }^{\mathrm{g}}$ The Lander Medical Practice, Truro, Cornwall, UK
\end{abstract}

\section{Keywords:}

comorbidity, mortality, multiple sclerosis

Received 7 January 2019

Accepted 10 July 2019

European Journal of

Neurology 2019, 0: 1-8

doi: $10.1111 /$ ene. 14040
Background and purpose: We aimed to determine the burden of comorbidities at the time of diagnosis of multiple sclerosis (MS), the risk of developing new comorbidities after diagnosis and the effect of comorbidities on mortality in patients with MS.

Methods: This study used data from 2526 patients with incident MS and 9980 age-, sex- and physician-matched controls without MS identified from the UK Clinical Practice Research Datalink.

Results: Before the MS diagnosis, the adjusted odds ratio for the association between MS and a Charlson comorbidity index score of $1-2,3-4$ or $\geq 5$ was 131 [95\% confidence interval (CI), 1.17-1.47], 1.65 (95\% CI, 1.20-2.26) or 3.26 (95\% CI, 1.58-6.70), respectively. MS was associated with increased risks of cardiovascular and neurological/mental diseases. After diagnosis, the adjusted hazard ratio for the association between MS and an increased risk of developing comorbidities was 1.13 (95\% CI, 1.00-1.29). The risk of developing any comorbidity in terms of neoplasms, musculoskeletal/connective tissue diseases or neurological/mental diseases was higher in MS. Patients with MS had a higher mortality risk compared with controls, with a hazard ratio of 2.29 (95\% CI, 1.81-2.73) after adjusting for comorbidities. There was a dose effect of pre-existing comorbidities on mortality.

Conclusions: Patients with MS have an increased risk of developing multiple comorbidities both before and after diagnosis and pre-existing comorbidities have an impact on survival.

\section{Introduction}

Multiple sclerosis (MS) is a chronic progressive disorder characterized by substantial disabilities that may impair daily activities [1], quality of life and survival [2]. MS-associated comorbidities at diagnosis may affect MS management [3] and increase disability

Correspondence: C. S. Constantinescu, Clinical Neurology Research Group, Division of Clinical Neuroscience, School of Medicine,

Queen's Medical Centre, University of Nottingham, Nottingham NG7 2UH, UK (tel.: + 44115 8231443; fax: + 44115 9709738; email: cris.constantinescu@nottingham.ac.uk).

*Joint senior author. severity [3]. For example, vascular comorbidity was associated with more rapid disability progression [4] and epilepsy contributed to a higher mortality risk [5]. Although the awareness of diverse comorbidities in patients with MS is growing [6,7], temporal changes in the comorbidity burden and the dose effect of comorbidity on survival risk have seldom been addressed.

This study examined the burden of comorbidities in patients with MS who were listed in the UK Clinical Practice Research Datalink (CPRD) and were diagnosed during 1997-2006. These patients and matched 
controls were followed to compare their subsequent accrual of comorbidities and all-cause mortality. We hypothesized, first, that patients with MS have higher risks of comorbidities at diagnosis and of developing comorbidities following diagnosis and, secondly, that the extent of comorbidity at MS diagnosis has a dose effect on survival.

\section{Methods}

\section{Data source}

The CPRD is a UK primary care database containing research-standard medical records prospectively collected from 1987 to 2016 (August 2016 version) [8]. A total of 701 general practitioner (GP) practices contributed in 2007, each caring for an average of 15963 patients. Our source population comprised all participants in England who contributed data to the CPRD between 1 January 1997 and 31 December 2006 and had valid linkage to secondary care data (Hospital Episode Statistics). The data were anonymized, eliminating the need for patient consent (approved protocol 14-070). CPRD records are well validated for diagnoses of diseases, including MS [9] and comorbidities [10]. This study included two parts: a matched case-control study to explore the associations of several pre-existing comorbidities with incident MS and a cohort study investigating the incidence of comorbidities during follow-up of patients with incident MS compared with subjects without MS.

\section{Definition of incident multiple sclerosis}

We used Read codes (Table S1) to identify patients with MS. The eligibility criteria for patients with incident MS were (i) no evidence of MS prior to the time of diagnosis (index date) and (ii) at least a 3-year continuous CPRD registration prior to the index date [9]. Patients were excluded if they or their GPs were no longer registered in the CPRD. The definition of MS was validated (equivalent to International Classification of Diseases-10 (ICD) code 340.0 between 1993 and 2000) and found to have a positive predictive value of $91.8 \%$ [9].

\section{Selection of controls}

Each case was randomly matched with up to four controls without MS from among CPRD participants who were active during 1997-2006. The controls and cases were matched by birth year ( \pm 2 years), sex, registered GP practice and the first year of continuous registration ( \pm 2 years) (Fig. 1). The purpose of matching GP practice and first year of continuous registration was to control for possible confounding due to registration with a given GP practice. Each of the matched controls was assigned the index date of their matched patient with MS and required to have been registered for at least 3 years before the index date.

\section{Study period}

We defined two periods for assessing comorbidity: (i) the 10 years prior to diagnosis and (ii) the period from diagnosis to the earliest date of the occurrence of comorbidities, death, transfer out, last data collection time for the practice or end of study (30 June 2016), whichever came first (Fig. S1).

\section{Comorbidities of interest}

We classified the pre-specified comorbidities evaluated into nine categories: neoplasms, cardiovascular diseases, renal diseases, metabolic and endocrine diseases, gastrointestinal and hepatic diseases, chronic pulmonary diseases, musculoskeletal and connective tissue diseases, neurological and mental disorders, and other [11]. To examine the burden of comorbidity in patients with MS compared with controls, we used the Charlson comorbidity index (CCI) (Table S2) [12]. The CCI is used to measure individual health status and comorbidity, and to control for confounding factors when predicting mortality [13]. We first categorized the CCI scores as $0,1-2,3-4$ or $\geq 5$ and then dichotomized the values as 0 or $\geq 1$.

\section{All-cause mortality}

Mortality and date of death were based on records in the main CPRD database. The CPRD employs an algorithm to identify the death dates of the participants. It was reported previously [14] that the validity of the recorded date of death in the CPRD has a sensitivity of 0.99 and specificity of 0.99 . The difference in the date of death between that in the CPRD and registration of death was $\leq 3$ months in $98.5 \%$ of deceased patients.

\section{Covariates}

Information on demographic factors (age and sex) and lifestyle factors (body mass index, smoking status and alcohol consumption) at diagnosis was considered in this study, as these are potentially modifiable factors affecting MS risk [15-17] and survival [18]. Patients received baseline health checks when they first registered with a GP practice, where the data 
Patients with MS in period 1997-2006 ( $n=2531)$

Continuous registration for $\geq 3$-years prior to the index date
Individuals with no evidence of MS in period 1997-2006

( $n=9335$ 073)

1. We selected people with continuous registration for $\geq 3$ years prior to the index date.

2. MS: controls at 1:4 ratio and matched by year of birth, sex, general practice (GP) and year of first continuous registration at the GP's practice

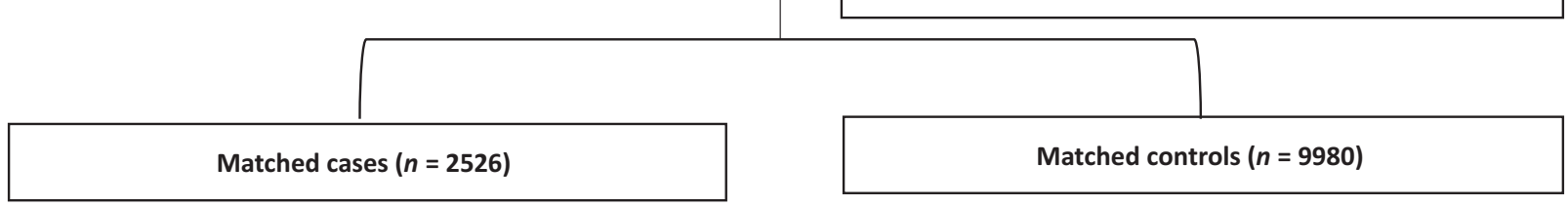

Figure 1 Flowchart of eligible patients with multiple sclerosis (MS) and controls.

were collected and recorded by nurses using standard protocols; body mass index $\left(\mathrm{kg} / \mathrm{m}^{2}\right)$ was calculated from these data.

\section{Statistical analysis}

For the matched case-control study, the prevalence of each comorbidity was calculated using the number of subjects ever diagnosed with a given comorbidity during the 10-year time frame before the index date as the numerator and the number of patients with incident MS or matched controls as the denominator. To estimate the association between MS and each comorbidity, we used conditional logistic regression analysis to calculate odds ratios (ORs), adjusting for lifestyle factors. Missing data were coded as 'unknown'. Considering the variability in follow-up time among the subjects before the index date, we conducted a sensitivity analysis by changing the period to 3 years.

For the cohort study of incident MS, designed to investigate the incidence of comorbidities, we followed the patients from the date of diagnosis to the first recorded diagnosis of a comorbidity and this interval was defined as the follow-up time. We plotted Kaplan-Meier curves of the cumulative probability of the occurrence of a new Charlson comorbidity. In individuals with no Charlson comorbidity at the index date, the cumulative probabilities of a new Charlson comorbidity were estimated at 1, 2, 5 and 10 years. Only patients without a comorbidity at the index date were considered in the estimation of hazard ratios (HRs) for a specific comorbidity using the Cox proportional hazards model. The HRs were adjusted by lifestyle factors as well as the matched variables at the index date.

We plotted Kaplan-Meier curves of the cumulative probability of the occurrence of all-cause mortality for patients with MS and controls, adjusting for covariates and the CCI score at the index date. We further limited our study to patients with incident MS to evaluate the relationship between the CCI score at diagnosis and all-cause mortality using Cox proportional hazards models adjusted for lifestyle factors and matched variables at the index date.

The Cox proportional hazards models employed in this study were tested for the assumption of proportionality by examining the log-log plot. The statistical analyses were conducted using SAS software (version 9.4, Cary, NC, USA).

\section{Results}

We identified 2526 patients with incident MS from 1997 to 2006 with a mean age of $45.0 \pm 12.4$ years; $71 \%$ were female. The median lengths of observation (interquartile range) before and after the index date were $12(7-21)$ and 10 (5-13) years, respectively. We followed 9980 controls with similar age and sex distributions and similar observation periods before and after the index date (Table 1). 
Table 1 Baseline characteristics of patients with incident multiple sclerosis (MS) and controls

\begin{tabular}{|c|c|c|c|}
\hline & $\begin{array}{l}\text { Incident MS } \\
(n=2526)\end{array}$ & $\begin{array}{l}\text { Controls } \\
(n=9980)\end{array}$ & $\begin{array}{l}\text { Adjusted ORs } \\
(95 \% \mathrm{CI})\end{array}$ \\
\hline Age (years) & $45.03 \pm 12.37$ & $45.17 \pm 12.43$ & \\
\hline \multicolumn{4}{|l|}{ Sex } \\
\hline Men & $736(29.14)$ & $2910(29.16)$ & \\
\hline Women & $1790(70.86)$ & $7070(70.84)$ & \\
\hline \multicolumn{4}{|c|}{ Observation (years) } \\
\hline $\begin{array}{l}\text { Prior to } \\
\text { index date }\end{array}$ & $12(7-21)$ & $12(7-20)$ & \\
\hline $\begin{array}{l}\text { After index } \\
\text { date }\end{array}$ & $10(5-13)$ & $10(6-13)$ & \\
\hline \multicolumn{4}{|l|}{ BMI $\left(\mathrm{kg} / \mathrm{m}^{2}\right)$} \\
\hline$<18.5$ & $983(38.92)$ & $3452(34.59)$ & $1.44(1.07-1.93)^{*}$ \\
\hline $18.5-24.9$ & $69(2.73)$ & $169(1.69)$ & Reference \\
\hline $25.0-29.9$ & $685(27.12)$ & $2670(26.75)$ & $0.90(0.80-1.00)$ \\
\hline$\geq 30$ & $469(18.57)$ & $1871(18.75)$ & $0.88(0.77-0.99)$ \\
\hline Unknown & $320(12.67)$ & $1818(18.22)$ & $0.58(0.50-0.68)$ \\
\hline \multicolumn{4}{|l|}{ Smoking } \\
\hline Non-smoker & $720(28.50)$ & $2953(29.59)$ & Reference \\
\hline $\begin{array}{l}\text { Current } \\
\text { smoker }\end{array}$ & $554(21.93)$ & $1542(15.45)$ & $1.53(1.34-1.75)^{*}$ \\
\hline Ex-smoker & $878(34.76)$ & $3261(32.68)$ & $1.09(0.97-1.24)$ \\
\hline Unknown & $374(14.81)$ & $2224(22.28)$ & $0.62(0.54-0.72)$ \\
\hline \multicolumn{4}{|c|}{ Alcohol consumption (units/week) } \\
\hline $\begin{array}{l}\text { Never/ } \\
\text { ex-drinker }\end{array}$ & $333(13.18)$ & $1085(10.87)$ & Reference \\
\hline Current 1-9 & $1321(52.30)$ & $4814(48.24)$ & $0.90(0.99-1.08)$ \\
\hline Current $\geq 10$ & $268(10.61)$ & $1030(10.32)$ & $0.83(0.68-1.00)^{*}$ \\
\hline Unknown & $604(23.91)$ & $3051(30.57)$ & $0.59(0.50-0.70)^{*}$ \\
\hline
\end{tabular}

BMI, body mass index; CI, confidence interval; OR, odds ratio. ${ }^{*} P<0.05$. Data are given as median (interquartile range), $n(\%)$ and mean $\pm \mathrm{SD}$.

\section{Retrospective observation}

At the index date, $22.7 \%$ of patients with MS had at least one Charlson comorbidity, compared with $16.8 \%$ of controls $(P<0.001)$. Patients with incident MS tended to have higher CCI scores than those of the controls (Table 2). Other neurological conditions were 10 -fold more common in patients with MS at 10 years before the MS diagnosis than in matched controls. Peptic ulcer [OR, 1.60; 95\% confidence interval (CI), 1.02-2.50], depression (OR, 1.54; 95\% CI, 1.38-1.72) and cardiovascular diseases (OR, 1.18; 95\% CI, 1.02-1.37) were also more common in patients with MS. The sensitivity analysis using a 3year time frame before the index date yielded similar results (Table $\mathrm{S} 3$ ).

In the analysis based on a 1 -year time frame, conditions more common in patients with incident MS than in controls included cerebrovascular diseases (OR, 11.09; 95\% CI, 5.74-21.4), neurological and mental disorders (OR, 3.60; 95\% CI, 3.11-4.17), peripheral vascular diseases (OR, 3.30; 95\% CI, 1.05-10.4), myocardial infarction (OR, 3.07; 95\% CI, 1.00-9.43), hyperlipidaemia (OR, 1.93; 95\% CI, 1.16-3.19) and anaemia (OR, 1.81; 95\% CI, 1.25-2.63).

\section{Follow-up data after the index date}

Table S4 shows the cumulative probability of any (or a specific) comorbidity after MS diagnosis. Among those with a CCI score of 0 at the index date, the cumulative probabilities of having a new Charlson comorbidity at 1 , 2, 5 and 10 years from the index date were $2.31 \%$ (number of individuals with a new comorbidity/number at risk at that time point, 57/2479), 4.16\% (43/1755), $8.57 \%(92 / 1539)$ and $17.23 \%(140 / 1139)$ in patients with incident MS, compared with 1.64\% (160/9807), 3.10\% (136/7621), 7.83\% (404/6881) and 15.78\% (543/5250) in the controls (Fig. S2, $P=0.2333$ ).

The adjusted HR $(95 \% \mathrm{CI})$ for having a CCI score $\geq 1$ in patients with MS was 1.13 (1.00-1.29). Table 3 shows that patients with MS compared with the controls had a higher risk of developing an incident comorbidity, including psychosis (adjusted HR, 2.36; 95\% CI, 1.34-4.16), depression (adjusted HR, 2.32; 95\% CI, 2.04-2.64) and malignancy (adjusted HR, $1.21 ; 95 \%$ CI, 1.00-1.46). Patients with MS had a lower risk of gout (adjusted HR, 0.59; 95\% CI, 0.370.94) compared with controls.

Figure 2 shows the survival curves for patients with MS and controls. All-cause mortality rates at 5 and 10 years after the index date were $5.11 \%$ and $10.27 \%$, respectively, in patients with incident MS, compared with $2.09 \%$ and $5.08 \%$ in controls (log-rank test, $P<0.0001)$ (Table S5). The crude and adjusted HRs for all-cause mortality in patients with MS were $2.38(95 \%$ CI, 2.02-2.81) and 2.29 (1.81-2.73), respectively (Table S6).

Next, we evaluated the association between the baseline CCI score and all-cause mortality in patients with MS. The 5-year all-cause mortality rates in patients with MS were $3.67 \%, 8.22 \%$ and $12.45 \%$ for patients with CCI scores of 0,1 and $\geq 2$ at the index date, respectively (log-rank test, $P<0.001$ ) (Fig. 3). After adjusting for lifestyle factors and matched variables at diagnosis, CCI scores of 1 and $\geq 2$ were associated with all-cause mortality (adjusted HR, 1.94; 95\% CI, 1.36-2.75; adjusted HR, 3.27; 95\% CI, 2.424.42 , respectively).

\section{Discussion}

At MS diagnosis, the respective prevalences of cerebrovascular diseases, neurological and mental disorders, peripheral vascular diseases, myocardial infarction, hyperlipidaemia and anaemia were 
Table 2 Comorbidities present 10 years before the index date

\begin{tabular}{|c|c|c|c|c|}
\hline & \multicolumn{4}{|c|}{ 10-year period before the index date } \\
\hline & Cases & Controls & Unadjusted OR & Adjusted $\mathrm{OR}^{\mathrm{a}}$ \\
\hline \multicolumn{5}{|l|}{ Charlson index } \\
\hline 0 & $1952(77.28)$ & $8303(83.2)$ & Reference & Reference \\
\hline $1-2$ & $501(19.83)$ & $1518(15.21)$ & $1.43(1.27-1.60)^{*}$ & $1.31(1.17-1.47)^{*}$ \\
\hline $3-4$ & $59(2.34)$ & $141(1.41)$ & $1.82(1.33-2.49)^{*}$ & $1.65(1.20-2.26)^{*}$ \\
\hline$\geq 5$ & $14(0.55)$ & $18(0.18)$ & $3.48(1.70-7.12)^{*}$ & $3.26(1.58-6.70)^{*}$ \\
\hline Neoplasms & $64(2.53)$ & $226(2.26)$ & $1.11(0.83-1.47)$ & $1.08(0.81-1.45)$ \\
\hline Solid malignancy, leukaemia and lymphoma & $61(2.41)$ & $210(2.1)$ & $1.15(0.86-1.54)$ & $1.12(0.84-1.51)$ \\
\hline Metastatic solid tumours & $4(0.16)$ & $7(0.07)$ & $2.00(0.57-7.01)$ & $1.96(0.56-6.91)$ \\
\hline Cardiovascular diseases & $325(12.87)$ & $1065(10.67)$ & $1.27(1.10-1.46)^{*}$ & $1.18(1.02-1.37)^{*}$ \\
\hline Hypertension & $222(8.79)$ & $867(8.69)$ & $1.00(0.85-1.18)$ & $0.93(0.79-1.10)$ \\
\hline Cardiac arrhythmias & $34(1.35)$ & $84(0.84)$ & $1.65(1.10-2.47)^{*}$ & $1.60(1.06-2.41)^{*}$ \\
\hline Cerebrovascular disease & $71(2.81)$ & $94(0.94)$ & $3.18(2.31-4.38)^{*}$ & $2.98(2.15-4.12)^{*}$ \\
\hline Congestive heart failure & $13(0.51)$ & $24(0.24)$ & $2.01(1.01-4.01)^{*}$ & $1.96(0.97-3.95)$ \\
\hline Myocardial infarction & $23(0.91)$ & $60(0.6)$ & $1.55(0.95-2.54)$ & $1.37(0.84-2.26)$ \\
\hline Peripheral vascular disease & $20(0.79)$ & $47(0.47)$ & $1.68(0.99-2.85)$ & $1.48(0.87-2.53)$ \\
\hline Valvular heart disease & $9(0.36)$ & $32(0.32)$ & $1.13(0.54-2.37)$ & $1.10(0.52-2.31)$ \\
\hline Genitourinary diseases & $18(0.71)$ & $79(0.79)$ & $0.89(0.53-1.49)$ & $0.84(0.50-1.41)$ \\
\hline Urolithiasis & $10(0.4)$ & $48(0.48)$ & $0.83(0.42-1.65)$ & $0.77(0.39-1.53)$ \\
\hline Renal diseases & $8(0.32)$ & $32(0.32)$ & $0.95(0.44-2.08)$ & $0.93(0.42-2.03)$ \\
\hline Metabolic and endocrine diseases & $208(8.23)$ & $696(6.97)$ & $1.20(1.01-1.42)^{*}$ & $1.10(0.92-1.30)$ \\
\hline Uncomplicated diabetes mellitus & $67(2.65)$ & $250(2.51)$ & $1.04(0.79-1.37)$ & $0.94(0.71-1.24)$ \\
\hline Diabetes mellitus with complications & $20(0.79)$ & $44(0.44)$ & $1.77(1.04-3.01)^{*}$ & $1.56(0.91-2.67)$ \\
\hline Hyperlipidaemia & $72(2.85)$ & $255(2.56)$ & $1.12(0.85-1.48)$ & $1.01(0.76-1.33)$ \\
\hline Hypothyroidism & $84(3.33)$ & $260(2.61)$ & $1.28(0.99-1.65)$ & $1.20(0.93-1.56)$ \\
\hline Gastrointestinal and hepatic diseases & $37(1.46)$ & $90(0.9)$ & $1.68(1.14-2.49)^{*}$ & $1.58(1.06-2.36)^{*}$ \\
\hline Peptic ulcer disease & $29(1.15)$ & $69(0.69)$ & $1.72(1.10-2.69)^{*}$ & $1.60(1.02-2.50)^{*}$ \\
\hline Mild liver disease & $7(0.28)$ & $18(0.18)$ & $1.57(0.65-3.81)$ & $1.49(0.61-3.66)$ \\
\hline Moderate to severe liver disease & $3(0.12)$ & $7(0.07)$ & $1.71(0.44-6.63)$ & $2.08(0.53-8.19)$ \\
\hline Chronic pulmonary diseases & $290(11.48)$ & $970(9.72)$ & $1.21(1.05-1.39)^{*}$ & $1.09(0.95-1.26)$ \\
\hline Musculoskeletal and connective tissue diseases & $148(5.86)$ & $509(5.1)$ & $1.15(0.94-1.39)$ & $1.06(0.87-1.29)$ \\
\hline Osteoarthritis & $63(2.49)$ & $260(2.61)$ & $0.94(0.70-1.25)$ & $0.91(0.68-1.21)$ \\
\hline Rheumatological disease & $70(2.77)$ & $282(2.83)$ & $0.95(0.72-1.24)$ & $0.86(0.65-1.13)$ \\
\hline Gout & $16(0.63)$ & $81(0.81)$ & $0.79(0.46-1.35)$ & $0.76(0.44-1.31)$ \\
\hline Neurological and mental disorders & $882(34.92)$ & $1783(17.87)$ & $2.65(2.39-2.93)^{*}$ & $2.49(2.24-2.76)^{*}$ \\
\hline Dementia & $0(0)$ & $5(0.05)$ & n.a. & n.a. \\
\hline Other neurological diseases & $297(11.76)$ & $119(1.19)$ & $11.3(9.00-14.2)^{*}$ & $10.72(8.52-13.5)^{*}$ \\
\hline Psychosis & $14(0.55)$ & $31(0.31)$ & $1.81(0.96-3.40)$ & $1.70(0.90-3.22)$ \\
\hline Depression & $616(24.39)$ & $1683(16.86)$ & $1.67(1.49-1.86)^{*}$ & $1.54(1.38-1.72)^{*}$ \\
\hline Other comorbidities & $252(9.98)$ & $871(8.73)$ & $1.16(1.00-1.35)^{*}$ & $1.10(0.94-1.28)$ \\
\hline Anaemia & $210(8.31)$ & $671(6.72)$ & $1.27(1.07-1.50)^{*}$ & $1.19(1.01-1.41)^{*}$ \\
\hline Psoriasis & $48(1.9)$ & $211(2.11)$ & $0.90(0.66-1.24)$ & $0.88(0.64-1.21)$ \\
\hline HIV infection & $0(0)$ & $2(0.02)$ & n.a. & n.a. \\
\hline
\end{tabular}

Charlson index includes myocardial infarction, congestive heart failure, peripheral vascular disease, cerebrovascular disease, dementia, chronic pulmonary disease, rheumatologic disease, peptic ulcer disease, mild liver disease, moderate/severe liver disease, diabetes mellitus (DM), DM with chronic complications, renal diseases, any malignancy (including leukaemia and lymphoma), metastatic solid tumour and human immunodeficiency virus (HIV) infection. n.a., not applicable; OR, odds ratio. ${ }^{a}$ Adjusted for body mass index class, smoking status and alcohol consumption. ${ }^{*} P<0.05$. Data are given as median (interquartile range) and $n(\%)$.

significantly higher than those in controls. After diagnosis, patients with MS were more likely than controls to develop psychosis, depression and malignancy. Although the Charlson comorbidity burden was already higher at the time of MS diagnosis, the risk of new comorbid conditions in patients after the diagnosis of incident MS did not differ from that in controls. These comorbidities at diagnosis had a dose effect on the survival of patients with MS. However, MS itself was associated with increased mortality independent of comorbidities. Comorbidity is not uncommon in patients with MS and the accumulation of comorbidities accompanying MS over time complicates management of the disease. Therefore, a good understanding of comorbidities in MS is needed to improve health outcomes. 
Table 3 Risk of incident comorbidity after the diagnosis of multiple sclerosis (MS) compared with controls without MS

\begin{tabular}{|c|c|c|c|c|}
\hline & MS cases & Controls without MS & Unadjusted HRs & Adjusted $\mathrm{HRs}^{\mathrm{a}}$ \\
\hline Charlson index $\geq 1$ & $1952(20.34)$ & $8303(18.85)$ & $1.19(1.05-1.34)^{\mathrm{a}}$ & $1.13(1.00-1.29)^{\mathrm{a}}$ \\
\hline Any increase in Charlson index & $2526(15.72)$ & $9980(15.68)$ & $1.08(0.96-1.22)$ & $1.05(0.92-1.19)$ \\
\hline Neoplasms & $2462(7.27)$ & $9754(6.55)$ & $1.24(1.03-1.50)^{\mathrm{a}}$ & $1.22(1.01-1.48)^{\mathrm{a}}$ \\
\hline Solid malignancy, leukaemia and lymphoma & $2464(7.22)$ & $9763(6.59)$ & $1.23(1.02-1.48)^{\mathrm{a}}$ & $1.21(1.00-1.46)^{\mathrm{a}}$ \\
\hline Metastatic solid tumours & $2522(0.59)$ & $9973(0.74)$ & $0.79(0.44-1.41)$ & $0.73(0.40-1.32)$ \\
\hline Cardiovascular diseases & $2201(15.77)$ & $8915(17.52)$ & $1.00(0.87-1.13)$ & $0.97(0.84-1.11)$ \\
\hline Hypertension & $2304(11.46)$ & $9113(14.38)$ & $0.91(0.78-1.05)$ & $0.89(0.76-1.03)$ \\
\hline Cardiac arrhythmias & $2492(1.97)$ & $9896(2.33)$ & $0.98(0.70-1.36)$ & $0.97(0.69-1.36)$ \\
\hline Cerebrovascular disease & $2455(3.01)$ & $9886(2.83)$ & $1.12(0.85-1.47)$ & $1.08(0.81-1.44)$ \\
\hline Congestive heart failure & $2513(0.76)$ & $9956(0.92)$ & $1.01(0.61-1.67)$ & $1.00(0.61-1.65)$ \\
\hline Myocardial infarction & $2503(1.28)$ & $9920(1.32)$ & $1.09(0.72-1.65)$ & $1.04(0.67-1.61)$ \\
\hline Peripheral vascular disease & $2506(1.00)$ & $9933(0.94)$ & $1.32(0.82-2.13)$ & $1.42(0.84-2.39)$ \\
\hline Valvular heart disease & $2517(0.87)$ & $9948(0.71)$ & $1.41(0.87-2.28)$ & $1.32(0.79-2.21)$ \\
\hline Genitourinary diseases & $2508(1.59)$ & $9901(1.55)$ & $1.08(0.77-1.52)$ & $1.03(0.72-1.47)$ \\
\hline Urolithiasis & $2516(0.64)$ & $9932(0.43)$ & $1.47(0.83-2.63)$ & $1.52(0.81-2.85)$ \\
\hline Renal diseases & $2518(0.95)$ & $9948(1.14)$ & $0.90(0.58-1.38)$ & $0.83(0.53-1.31)$ \\
\hline Metabolic and endocrine diseases & $2318(11.69)$ & $9284(11.75)$ & $1.08(0.93-1.26)$ & $1.08(0.93-1.27)$ \\
\hline Uncomplicated diabetes mellitus & $2459(4.51)$ & $9730(4.44)$ & $1.10(0.88-1.38)$ & $1.08(0.84-1.40)$ \\
\hline Diabetes mellitus with complications & $2506(4.27)$ & $9936(4.58)$ & $1.07(0.84-1.37)$ & $1.03(0.78-1.35)$ \\
\hline Hyperlipidaemia & $2454(5.26)$ & $9725(5.68)$ & $1.03(0.82-1.29)$ & $1.00(0.80-1.26)$ \\
\hline Hypothyroidism & $2442(3.97)$ & $9720(3.72)$ & $1.07(0.85-1.35)$ & $1.03(0.81-1.30)$ \\
\hline Gastrointestinal and hepatic diseases & $2489(1.69)$ & $9890(1.32)$ & $1.43(0.99-2.07)$ & $1.39(0.94-2.05)$ \\
\hline Peptic ulcer disease & $2497(0.76)$ & $9911(0.61)$ & $1.47(0.85-2.54)$ & $1.45(0.82-2.55)$ \\
\hline Mild liver disease & $2519(0.87)$ & $9962(0.7)$ & $1.28(0.76-2.17)$ & $1.11(0.61-2.00)$ \\
\hline Moderate to severe liver disease & $2523(0.08)$ & $9973(0.14)$ & $0.62(0.14-2.85)$ & $1.08(0.27-4.29)$ \\
\hline Chronic pulmonary diseases & $2236(5.37)$ & $9010(6.57)$ & $0.86(0.70-1.06)$ & $0.83(0.67-1.03)$ \\
\hline Musculoskeletal and connective tissue diseases & $2378(10.26)$ & $9471(8.72)$ & $1.24(1.07-1.45)^{\mathrm{a}}$ & $1.20(1.02-1.40)^{\mathrm{a}}$ \\
\hline Osteoarthritis & $2463(4.51)$ & $9720(5.47)$ & $0.90(0.74-1.10)$ & $0.92(0.75-1.14)$ \\
\hline Rheumatological disease & $2456(3.46)$ & $9698(4.10)$ & $0.89(0.70-1.15)$ & $0.86(0.66-1.11)$ \\
\hline Gout & $2510(1.00)$ & $9899(1.72)$ & $0.64(0.42-0.97)$ & $0.59(0.37-0.94)$ \\
\hline Neurological and mental disorders & $1644(30.54)$ & $8197(15.08)$ & $2.61(2.28-2.99)^{\mathrm{a}}$ & $2.50(2.17-2.88)^{\mathrm{a}}$ \\
\hline Dementia & $2526(0.51)$ & $9975(0.51)$ & $1.12(0.56-2.25)$ & $1.28(0.66-2.49)$ \\
\hline Other neurological diseases & $2229(5.29)$ & $9861(1.53)$ & $4.33(3.21-5.83)^{\mathrm{a}}$ & $4.49(3.28-6.13)^{\mathrm{a}}$ \\
\hline Psychosis & $2512(1.00)$ & $9949(0.42)$ & $2.24(1.32-3.80)^{\mathrm{a}}$ & $2.36(1.34-4.16)^{\mathrm{a}}$ \\
\hline Depression & $1910(26.54)$ & $8297(13.49)$ & $2.43(2.14-2.76)^{\mathrm{a}}$ & $2.32(2.04-2.64)^{\mathrm{a}}$ \\
\hline Other comorbidities & $2274(8.62)$ & $9109(8.54)$ & $1.02(0.86-1.20)$ & $0.99(0.83-1.18)$ \\
\hline Anaemia & $2322(7.49)$ & $9331(7.35)$ & $1.01(0.85-1.21)$ & $0.99(0.81-1.19)$ \\
\hline Psoriasis & $2478(1.74)$ & $9769(1.73)$ & $1.13(0.79-1.62)$ & $1.18(0.80-1.73)$ \\
\hline HIV infection & $2526(0.00)$ & $9978(0.00)$ & n.a. & n.a. \\
\hline
\end{tabular}

Figures show the number in the at-risk population (percentage of the at-risk population who developed incident comorbidity) and hazard ratios (HRs) $\left(95 \%\right.$ confidence interval). ${ }^{\mathrm{a}}$ Adjusted for body mass index class, smoking status and alcohol consumption. ${ }^{\mathrm{b}} P<0.05$. HIV, human immunodeficiency virus; n.a., not applicable. Data are given as median (interquartile range) and $n(\%)$.

Several systematic reviews confirmed that comorbidities are common in patients with MS $[6,19,20]$. In general, however, previous studies did not evaluate the clinical course of MS relative to diagnosis. Consistent with previous research, this study found that cardiovascular diseases including cardiac arrhythmia, ischemic heart disease and congestive heart failure [7], mental disorders (particularly depression) [6] and probably certain types of cancer [7] seem more common in the MS population than in controls.

The relationships between MS and comorbidities are complex [21] and can be broadly classified as direct causal relationships, associated risk factors or heterogeneous effects [22]. For example, mental health problems in patients with MS are common [6]. Although the pathophysiology is not fully understood, both MS lesions and microstructural white matter changes may lead to mental health manifestations such as depression [23]. Other factors such as genetic and psychosocial factors may also be involved in the link between depression and MS [24]. Our results support associations between MS and psychiatric illnesses.

Associated comorbidities have implications for clinical management of MS [3]. First, the existence of comorbidities may complicate MS management. For 


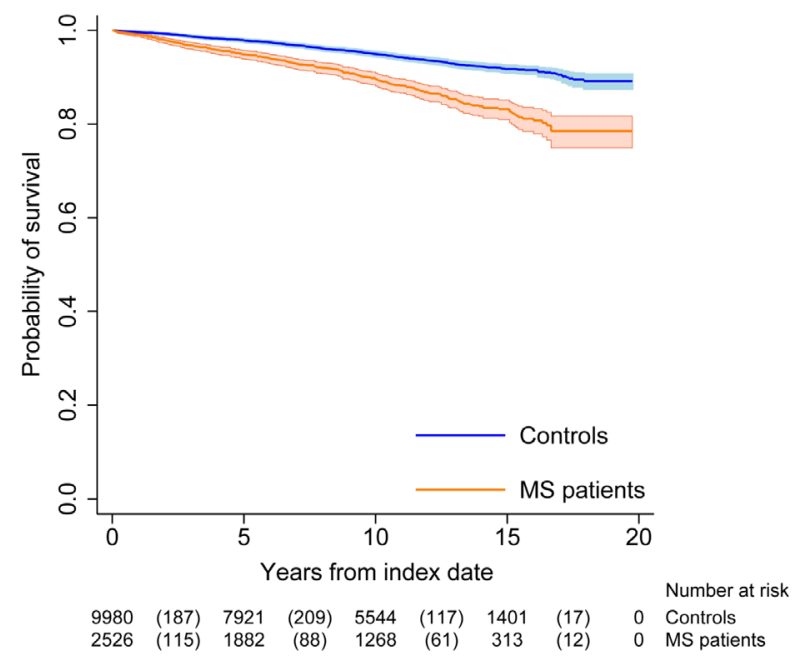

Figure 2 Survival curves for patients with incident multiple sclerosis (MS) and controls. After the index date, cumulative mortality from any cause was higher in patients with incident MS (red) than in controls (blue) (log-rank test, $P<0.0001$ ).

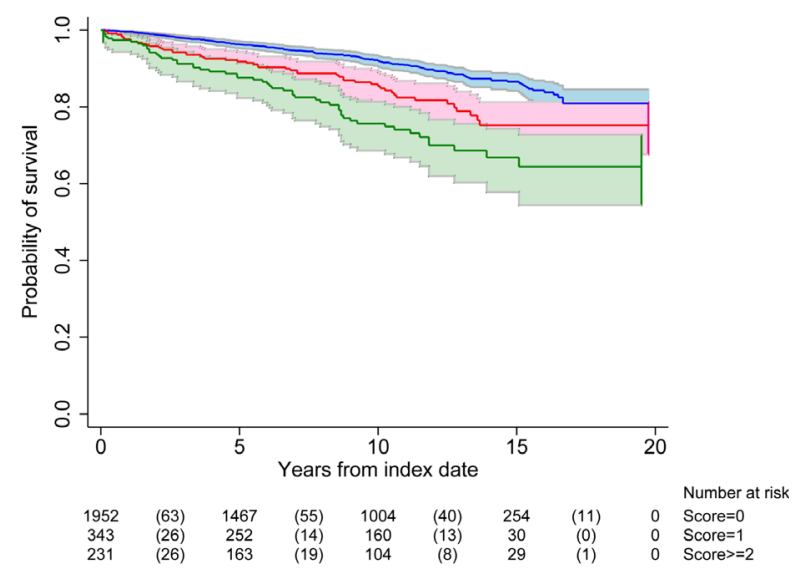

Figure 3 Survival curve for patients with incident multiple sclerosis (MS) with a Charlson comorbidity index (CCI) of 1 or $\geq 2$ at diagnosis compared with those without comorbidities (CCI of 0 ). After the index date, cumulative mortality was higher in patients with incident MS with a pre-existing CCI of 1 (red) or $\geq 2$ (green) compared with a score of 0 (blue) (log-rank test, $P<0.0001)$.

example, fingolimod, a medication commonly used to treat MS, is contraindicated in patients with recent cardiovascular problems [25]. Secondly, an assessment of comorbidities at diagnosis and during follow-up seems warranted, as one-third of the patients with MS in this study developed a comorbidity within 5 years of diagnosis. Thirdly, a comorbidity index can be used to estimate prognosis by considering the overall comorbidity burden and its effects on mortality risk in MS.
Three main limitations of this study need to be discussed. First, misclassification bias may exist for MS and comorbidities, given that physician diagnoses were used as case definitions. However, the validity of the diagnoses of MS and most comorbidities in the CPRD has been validated $[9,10]$. With identical case definitions for all patients, the potential for differential misclassification should be minimized. Secondly, the possibility of ascertainment bias between patients with incident MS and controls cannot be excluded entirely. The nature of healthcare utilization may lead to a higher probability of identifying comorbidities in regular users of healthcare services. Thirdly, some potential confounders such as ethnicity, education, annual household income and treatment methods were not considered.

\section{Conclusions}

Patients with MS have increased risks of developing multiple comorbidities both before and after diagnosis and pre-existing comorbidities have a dose effect on survival.

\section{Acknowledgements}

This work was funded by Chang Gung Memorial Hospital (CMRPG4A0121-0124, CMRPG3G16911692, CORPG3G0441, CORPG3G0481) and University of Nottingham.

\section{Disclosure of conflicts of interest}

The authors declare no financial or other conflicts of interest.

\section{Supporting Information}

Additional Supporting Information may be found in the online version of this article:

Table S1. Read codes for multiple sclerosis.

Table S2. Charlson comorbidity index scores.

Table S3. Comorbidities present 3 years before the index date.

Table S4. Cumulative probabilities of comorbidities after diagnosis of multiple sclerosis.

Table S5. Cumulative probabilities of all-cause mortality after diagnosis of multiple sclerosis.

Table S6. Predictors of all-cause mortality. 
Figure S1. Observation of comorbidities in a patient with incident multiple sclerosis.

Figure S2. The cumulative probabilities of developing a new Charlson comorbidity in patients with multiple sclerosis (MS) (red) and matched controls without MS (blue) who had a Charlson comorbidity index score of 0 at the index date.

\section{References}

1. Buzaid A, Dodge MP, Handmacher L, Kiltz PJ. Activities of daily living: evaluation and treatment in persons with multiple sclerosis. Phys Med Rehabil Clin $N$ Am 2013; 24: 629-638.

2. Manouchehrinia A, Tanasescu R, Tench CR, Constantinescu CS. Mortality in multiple sclerosis: meta-analysis of standardised mortality ratios. J Neurol Neurosurg Psychiatry 2016; 87: 324-331.

3. Marrie RA. Comorbidity in multiple sclerosis: implications for patient care. Nat Rev Neurol 2017; 13: 375-382.

4. Marrie RA, Rudick R, Horwitz R, et al. Vascular comorbidity is associated with more rapid disability progression in multiple sclerosis. Neurology 2010; 74: 10411047.

5. Chou IJ, Kuo CF, Tanasescu R, et al. Epilepsy and associated mortality in patients with multiple sclerosis. Eur J Neurol 2019; 26: 342-e23.

6. Marrie RA, Reingold S, Cohen J, et al. The incidence and prevalence of psychiatric disorders in multiple sclerosis: a systematic review. Mult Scler 2015; 21: 305-317.

7. Jick SS, Li L, Falcone GJ, Vassilev ZP, Wallander MA. Mortality of patients with multiple sclerosis: a cohort study in UK primary care. $J$ Neurol 2014; 261: 1508-1517.

8. Walley $\mathrm{T}$, Mantgani A. The UK general practice research database. Lancet 1997; 350: 1097-1099.

9. Hernan MA, Jick SS, Olek MJ, Jick H. Recombinant hepatitis B vaccine and the risk of multiple sclerosis: a prospective study. Neurology 2004; 63: 838-842.

10. Herrett E, Thomas SL, Schoonen WM, Smeeth L, Hall AJ. Validation and validity of diagnoses in the General Practice Research Database: a systematic review. $\mathrm{Br} J$ Clin Pharmacol 2010; 69: 4-14.

11. Feinstein AR. The pre-therapeutic classification of comorbidity in chronic disease. J Chronic Dis 1970; 23: 455-468.

12. Charlson ME, Pompei P, Ales KL, MacKenzie CR. A new method of classifying prognostic comorbidity in longitudinal studies: development and validation. $J$ Chronic Dis 1987; 40: 373-383.

13. Peterson JC, Paget SA, Lachs MS, Reid MC, Charlson ME. The risk of comorbidity. Ann Rheum Dis 2012; 71: 635-637.

14. Kuo CF, Grainge MJ, Mallen C, Zhang W, Doherty M. Comorbidities in patients with gout prior to and following diagnosis: case-control study. Ann Rheum Dis 2016; 75: $210-217$.

15. Hedstrom AK, Hillert J, Olsson T, Alfredsson L. Alcohol as a modifiable lifestyle factor affecting multiple sclerosis risk. JAMA Neurol 2014; 71: 300-305.

16. Hedstrom AK, Olsson T, Alfredsson L. High body mass index before age 20 is associated with increased risk for multiple sclerosis in both men and women. Mult Scler 2012; 18: 1334-1336.

17. Sundstrom P, Nystrom L, Hallmans G. Smoke exposure increases the risk for multiple sclerosis. Eur $J$ Neurol 2008; 15: 579-583.

18. Manouchehrinia A, Weston M, Tench CR, Britton J, Constantinescu CS. Tobacco smoking and excess mortality in multiple sclerosis: a cohort study. J Neurol Neurosurg Psychiatry 2014; 85: 1091-1095.

19. Edwards LJ, Constantinescu CS. A prospective study of conditions associated with multiple sclerosis in a cohort of 658 consecutive outpatients attending a multiple sclerosis clinic. Mult Scler 2004; 10: 575-581.

20. Marrie RA, Reider N, Cohen J, et al. A systematic review of the incidence and prevalence of cardiac, cerebrovascular, and peripheral vascular disease in multiple sclerosis. Mult Scler 2015; 21: 318-331.

21. Valderas JM, Starfield B, Sibbald B, Salisbury C, Roland M. Defining comorbidity: implications for understanding health and health services. Ann Fam Med 2009; 7: 357-363.

22. Rhee SH, Hewitt JK, Lessem JM, Stallings MC, Corley RP, Neale MC. The validity of the Neale and Kendler model-fitting approach in examining the etiology of comorbidity. Behav Genet 2004; 34: 251-265.

23. Feinstein A, O'Connor P, Akbar N, Moradzadeh L, Scott CJ, Lobaugh NJ. Diffusion tensor imaging abnormalities in depressed multiple sclerosis patients. Mult Scler 2010; 16: 189-196.

24. Feinstein A, Magalhaes S, Richard JF, Audet B, Moore C. The link between multiple sclerosis and depression. Nat Rev Neurol 2014; 10: 507-517.

25. Camm J, Hla T, Bakshi R, Brinkmann V. Cardiac and vascular effects of fingolimod: mechanistic basis and clinical implications. Am Heart J 2014; 168: 632-644. 